\title{
Moderate physical activity may not decrease the risk of cardiovascular disease in persistently overweight and obesity adults
}

\author{
Qiuyue Tian ${ }^{1 \dagger}$, Biyan Wang ${ }^{1 \dagger}$, Shuohua Chen ${ }^{2}$, Shouling Wu 2* $^{*^{*}}$ and Youxin Wang ${ }^{1^{*}}$ (1)
}

\begin{abstract}
Background: Body mass index (BMI) and physical activity (PA) has been documented to be associated with cardiovascular disease (CVD). However, the evidences regarding joint phenotypes of BMI and PA trajectories with risk for CVD and all-cause mortality are still limited.
\end{abstract}

Methods: Participants from the Kailuan Study, followed up during 2006-2019 were included, with primary outcomes of CVDs (myocardial infarction or stroke) and all-cause mortality. BMI and PA were repeatedly measured at least three times, and thus joint phenotypes trajectory groups were identified by group-based trajectory modeling. Cox proportional hazards models were used to examine the associations between trajectory groups and CVDs and all-cause mortality.

Results: Totally 88,141 (6 trajectories) and 89,736 participants ( 5 trajectories) were included in the final analyses relating trajectories to CVDs and all-cause mortality, respectively. Compared with persistent normal-weight with moderate PA group, participants were associated with increased risk of CVD in persistent overweight with moderate PA trajectory group (adjusted hazard ratio [aHR]: 1.31, 95\% confidence interval [CI]: 1.22-1.41) and persistent obesity with moderate PA trajectory group (aHR: $1.55,95 \% \mathrm{Cl}: 1.41-1.69$ ). While the rising to overweight with moderate PA in normal-weight status with active PA (aHR: $0.72,95 \%$ Cl:0.65-0.79), persistent overweight with moderate PA (aHR: 0.92 , 95\% Cl:0.87-0.97) and decline to normal-weight in overweight status with moderate PA (aHR: 0.73, 95\% Cl:0.67-0.80) trajectories group were significantly associated with decreased all-cause mortality risk. The associations remained robust among stratifying by age and sex individuals and sensitive analysis.

Conclusions: The long-term trajectories analysis showed that moderate PA may not decrease the risk of CVD in persistently overweight and obesity adults.

Keywords: Cardiovascular disease, Body mass index, Physical activity, Long-term trajectories, All-cause mortality

*Correspondence: drwus@@163.com; wangy@ccmu.edu.cn

${ }^{\dagger}$ Qiuyue Tian and Biyan Wang contributed equally

1 Beijing Key Laboratory of Clinical Epidemiology, School of Public Health, Capital Medical University, 10 YouanmenXitoutiao, Beijing 100069, China

${ }^{2}$ Department of Cardiology, Kailuan General Hospital, North China University of Science and Technology, 57 Xinhua East Road, Tangshan 063000, China

\section{Background}

In the worldwide, cardiovascular diseases (CVDs) is the leading cause of mortality with a major contributor to disability, according to the Global Burden of Disease Study 2019 [1, 2]. CVDs, principally ischemic heart disease and stroke, account for $40 \%$ of the cause of deaths in the Chinese population [3]. The dramatic increase in obesity and overweight prevalence was caused by changes in dietary and physical activity patterns, especially during 
the past two decades of rapid economic growth in China $[4,5]$.

The relationship of obesity and CVDs has been widely discussed over recent years. Most epidemiological evidences supporting a link between obesity and CVDs are based on one time point assessment $[6,7]$. In addition, obesity was also significantly associated with higher allcause mortality [8, 9]. However, despite these adverse effects on increasing the risk of CVDs, numerous studies demonstrated the obesity paradox, where CVDs patients with overweight and obesity seem to have a survival advantage [10-12]. Previous research has highlighted the need to take into account the time-varying nature of obesity and changes in weight status over the lifespan to understand the true relationship of obesity and disease risk [13]. Although body mass index (BMI) is widely acceptable to evaluate weight status and related disease risk, a simple classification of overweight and obesity by $\mathrm{BMI}$ at one point time may not be sufficient to explain long-term weight. Moreover, considering longitudinal changes in obesity and their associations with CVDs and mortality may help direct us toward a more meaningful clinical question, because it provides insight as to whether intervening on the BMI pathway is in fact able to provide a survival benefit.

Physical activity (PA) is closely related to obesity, and any comprehensive study focused on obesity should take PA into account. Several studies have confirmed that PA is related to lower risk of CVDs and longer life expectancy $[14,15]$. In addition, studies also showed that participants with overweight or obesity but active PA were not associated with a higher CVDs risk [16, 17]. However, a recent study involving 527,662 participants showed that obesity or overweight was associated an increased risk of CVDs regardless of PA levels, which similar with several cohort studies [18-20]. Thus, it is necessary to investigate the association between long-term trajectory of joint PABMI phenotypes and outcomes.

In the study, we used the group-based trajectory model (GBTM) to identify distinct trajectories of joint phenotypes of obesity and PA and to examine the associations between these long-term variation trajectories and risks of CVDs (including myocardial infarction (MI) and stroke) and all-cause mortality.

\section{Methods}

\section{Study design and participants}

We performed a population-based retrospective longitudinal cohort study based on data from the Kailuan study, which is an ongoing prospective study in Tangshan, China. Briefly, from June 2006 to October 2007, 101,510 individuals (including 81,110 men and 20,400 women, aged $18-98$ years) in the Kailuan community were enrolled to participate in the study, and followed up biennially [21-24]. In this study, we identified the joint phenotypes trajectories using joint phenotypes status in 2006, 2008, 2010, 2012, 2014, 2016, and used these patterns to predict incident CVDs and all-cause mortality. Therefore, we excluded participants who did not have data for BMI (or height and weight) and PA at baseline, have baseline BMI less than $18.5 \mathrm{~kg} / \mathrm{m}^{2}$, and have history of CVDs at baseline. In addition, we further excluded participants with less than 3 times repeatedly measurement of joint BMI-PA phenotypes status due to loss to follow-up including occurring outcomes or other reasons during 2006 to 2016, respectively. This study was approved by the ethics committees of Kailuan General Hospital. Written informed consent form was obtained from all participants.

\section{Assessment of the joint phenotypes at baseline}

BMI was calculated as weight in kilograms divided by the square of height in meters. According to the Chinesespecific criteria, normal weight (NW) were defined as $18.5 \leq \mathrm{BMI}<24.0 \mathrm{~kg} / \mathrm{m}^{2}$, overweight (OW) were defined as $24.0 \leq \mathrm{BMI}<28.0 \mathrm{~kg} / \mathrm{m}^{2}$, and obesity were defined as $\mathrm{BMI} \geq 28 \mathrm{~kg} / \mathrm{m}^{2}$ [25]. Participants were divided into three groups: inactive ( $<1$ time/week), moderate (1-2 times/ week), and active ( $\geq 3$ times/week and $\geq 30 \mathrm{~min} /$ time) according to the self-reported the frequency of PA from questionnaire [26]. Then, at baseline participants were categorized into nine joint phenotypes groups: NW and active PA (NWAPA), NW and moderate PA (NWMPA), NW and inactive PA (NWIPA), OW and active PA (OWAPA), OW and moderate PA (OWMPA), OW and inactive PA (OWIPA), obesity and active PA (OAPA), obesity and moderate PA (OMPA), obesity and inactive PA (OIPA).

\section{Assessment of the joint phenotypes trajectories}

In this study, we evaluated the joint BMI-PA phenotypes status among participants from 2006 to 2016. On the basis of no less than three values of joint BMI-PA phenotypes status, we used the GBTM to identify subgroups, which shared similar underlying trajectories [27-29]. Model fit was assessed using the Akakike information criteria (AIC), Bayesian information criterion (BIC), and Average posterior probability (AvePP) [28]. We initiated a model with one trajectory and then compared the model fit index to that with two, three, four, five, six, seven, eight, and nine, respectively. Then, cubic, quadratic, and linear terms were considered and evaluated based on their significance level, starting with the highest polynomial. In our final model, 6 trajectories with cubic order terms and 5 trajectories with cubic order terms as 
the best fit were included in the further analyses relating trajectories to CVDs and all-cause mortality respectively.

\section{Follow-up and outcomes}

All participants were followed up every two years until death or December 31, 2019. The primary outcomes were CVDs (include stroke and MI) and all-cause mortality. The diagnoses information on CVDs was obtained from medical records from medical insurance or hospitals $[21,23]$. Death diagnosis information comes from family report, death certificates from provincial vital statistics offices, and medical records from medical insurance or hospitals $[21,23]$.

\section{Covariates}

Socio-demographic data, lifestyle factors and medical history were collected via standardized questionnaires and clinical examinations at baseline and follow-up [4, $21,23]$. We selected age, sex, types of work, seat time, walking instead of the elevators, educational level, smoking status, drinking status, family per-member monthly income, salt intake, drinking tea status, C-reactive protein (CRP), and history of diseases (hypertension, diabetes, and hyperlipidemia) as covariates. Each covariate was collected during biennially follow-up.

\section{Statistical analyses}

Continuous variables were described as median with interquartile range (IQR), and categorical variables were showed as number (percentage). The comparisons of continuous or categorical variables were conducted using the Kruskal-Wallis test or chi-square tests, respectively and the trend association was estimated using linear regression or Chi-square trend tests.

Person-years were calculated from the date of baseline examination to the date of CVDs or death, or the end of follow-up (December 31, 2019), whichever came first. The adjusted cumulative incidence of CVDs or all-cause mortality was estimated using Kaplan-Meier method and compared by log-rank. The proportional hazards assumption was tested by the Schoenfeld residuals, and no violation was found. Associations between trajectory groups and the risk of CVDs and all-cause mortality were estimated by cox proportional hazards regression models with 95\% confidence intervals (CIs). We fitted 3 models. Model 1 was a crude model without adjusted covariates. Model 2 was adjusted for age, sex, types of work, seat time, and walking instead of the elevators. Model 3 was further adjusted for educational level, smoking status, drinking status, family per-member monthly income, salt intake, drinking tea status, CRP, and history of diseases (hypertension, diabetes, and hyperlipidemia).
To test the robustness of the main results, several sensitivity analyses were performed. Considering non-CVDs death as a competing risk event rather than a censoring event, Fine-Gray competing risk model was applied to address this issue. Considering the time dependence of variables, time-dependent cox proportional hazards models were constructed to explore the associations between trajectory groups and the risk of CVDs and allcause mortality, while simultaneously adjusting for timevarying confounders and other covariates. Likelihood ratio test was conducted to examine statistical interactions among trajectory groups, sex, and age $(<65$ years and $\geq 65$ years) in association with CVDs and all-cause mortality by comparing $-2 \log$ likelihood chi-square between nested models, with or without the multiplication interaction terms.

All statistical analyses were conducted using SAS, version 9.4 (SAS Institute Inc). Two-sided $P<0.05$ was considered statistically significant.

\section{Results}

Of 101,510 participants were enrolled, 10,646 participants were excluded due to missing information on BMI or PA $(n=5659)$, history of stroke or MI $(n=3334)$, CVDs at baseline $(n=715)$, and BMI of less than $18.5 \mathrm{~kg} /$ $\mathrm{m}^{2}(n=3290)$. In addition, we excluded participants with less than 3 times repeatedly measurement of joint BMIPA phenotypes status due to loss to follow-up including occurring CVDs $(n=2723)$ or all-cause mortality ( $n=1128$ ) during 2006 to 2016, respectively (Fig. 1). Finally, the trajectories of joint BMI-PA phenotypes and their association with future CVDs and all-cause mortality risk were examined among the remaining 88,141 (accounting for $97 \%$ of samples) and 89,736 participants (accounting for $98.76 \%$ of samples), respectively.

We categorized the study population into six observed discrete trajectories to predict incident CVDs (Fig. 2a): $34.13 \%$ of participants who remained within the OWMPA range during 11 follow-up years (referred to as "persistent OW with moderate active physical activity (MPA)" group); $31.35 \%$ of the participants who remained within the NWMPA range during 11 follow-up years (referred to as "persistent NW with MPA" group); $13.46 \%$ of the participants who remained within the OMPA range during 11 follow-up years (referred to as "persistent obesity with MPA" group); $8.29 \%$ of participants who started with NWMPA status and experienced an increase from NW to OW with MPA (referred to as "rising to OW in NW status with MPA" group); $7.87 \%$ of the participants who started with OWMPA status and experienced an decrease from OW to NW with MPA (referred to as "decline to NW in OW status with MPA" group); and $4.90 \%$ of the participants who started with 


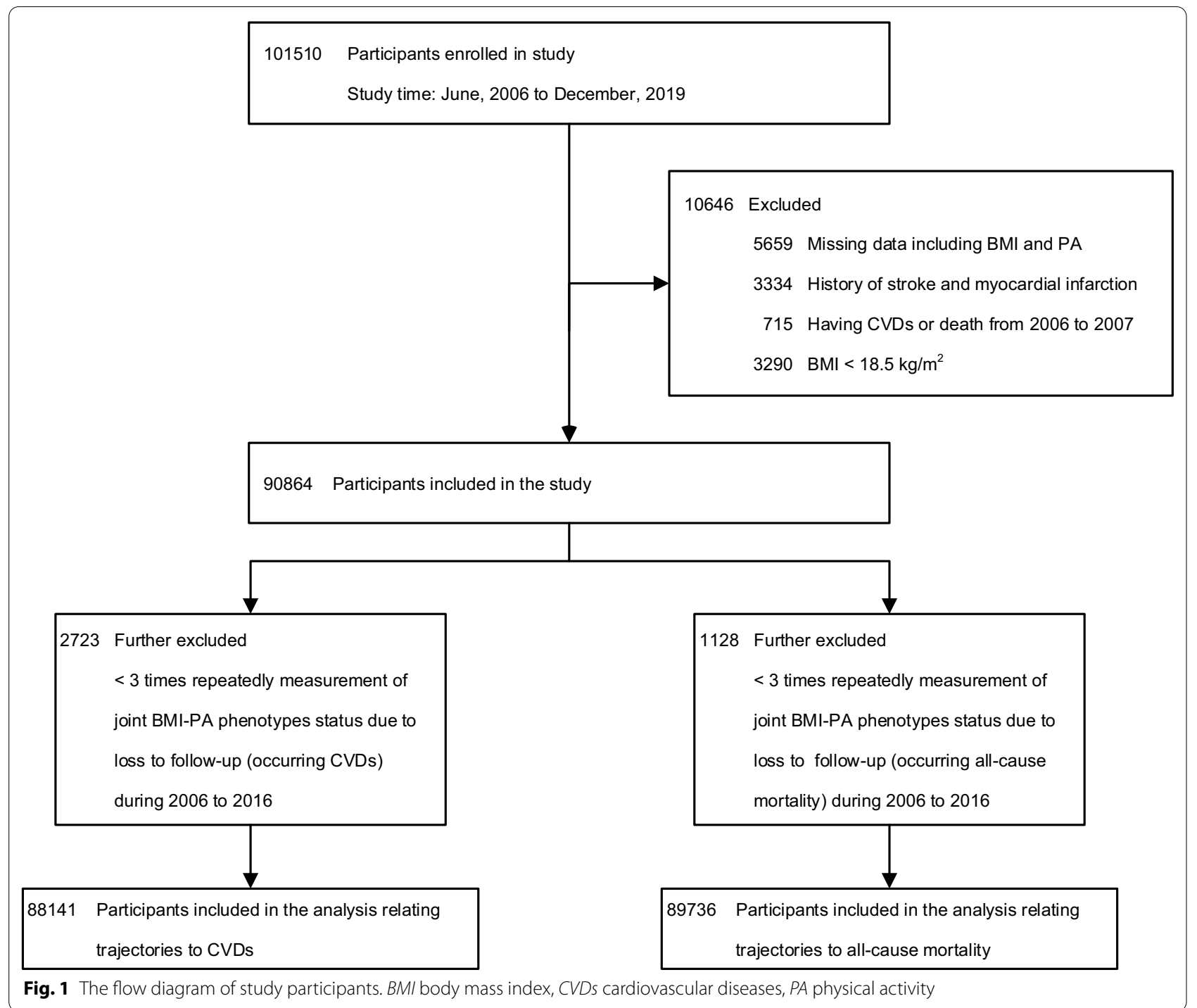

OMPA status and experienced an decrease from obesity to OW with MPA (referred to as "decline to OW in obesity status with MPA" group). Similarly, we categorized the study population into five observed discrete trajectories to predict all-cause mortality (Fig. 2b): $36.00 \%$ of participants who remained within the OWMPA range during 11 follow-up years (referred to as "persistent OW with MPA" group); $31.65 \%$ of participants who started with NWAPA status and experienced an increase from NW to OW and a decrease from active physical activity (APA) to MPA (referred to as "rising to OW with MPA in NW status with APA" group); $15.96 \%$ of the participants who remained within the OMPA range during 11 follow-up years (referred to as "persistent obesity with MPA" group); $8.75 \%$ of the participants who remained within the NWMPA range during 11 follow-up years (referred to as "persistent NW with MPA" group); 7.64\% of the participants who started with OWMPA status and experienced an decrease from OW to NW with MPA (referred to as "decline to NW in OW status with MPA" group). The baseline characteristics of participants according trajectory groups are presented in Additional file 1: Table S1, S2. Trajectory groups with obesity were more likely to be had higher proportion of older individuals, history of disease (hypertension, diabetes, and hyperlipidemia), and tea drinker, longer seat time, higher level of salt intake, lower high-density lipoprotein cholesterol (HDL-C), and higher blood pressure, waist circumference (WC), hip circumference $(\mathrm{HC})$, triglycerides (TG), total cholesterol (TC), fasting blood glucose (FBG), CRP, and low-density lipoprotein cholesterol (LDL-C), compared with other trajectory groups.

The unadjusted and adjusted cumulative incidence of CVDs and all-cause mortality according to the trajectory 


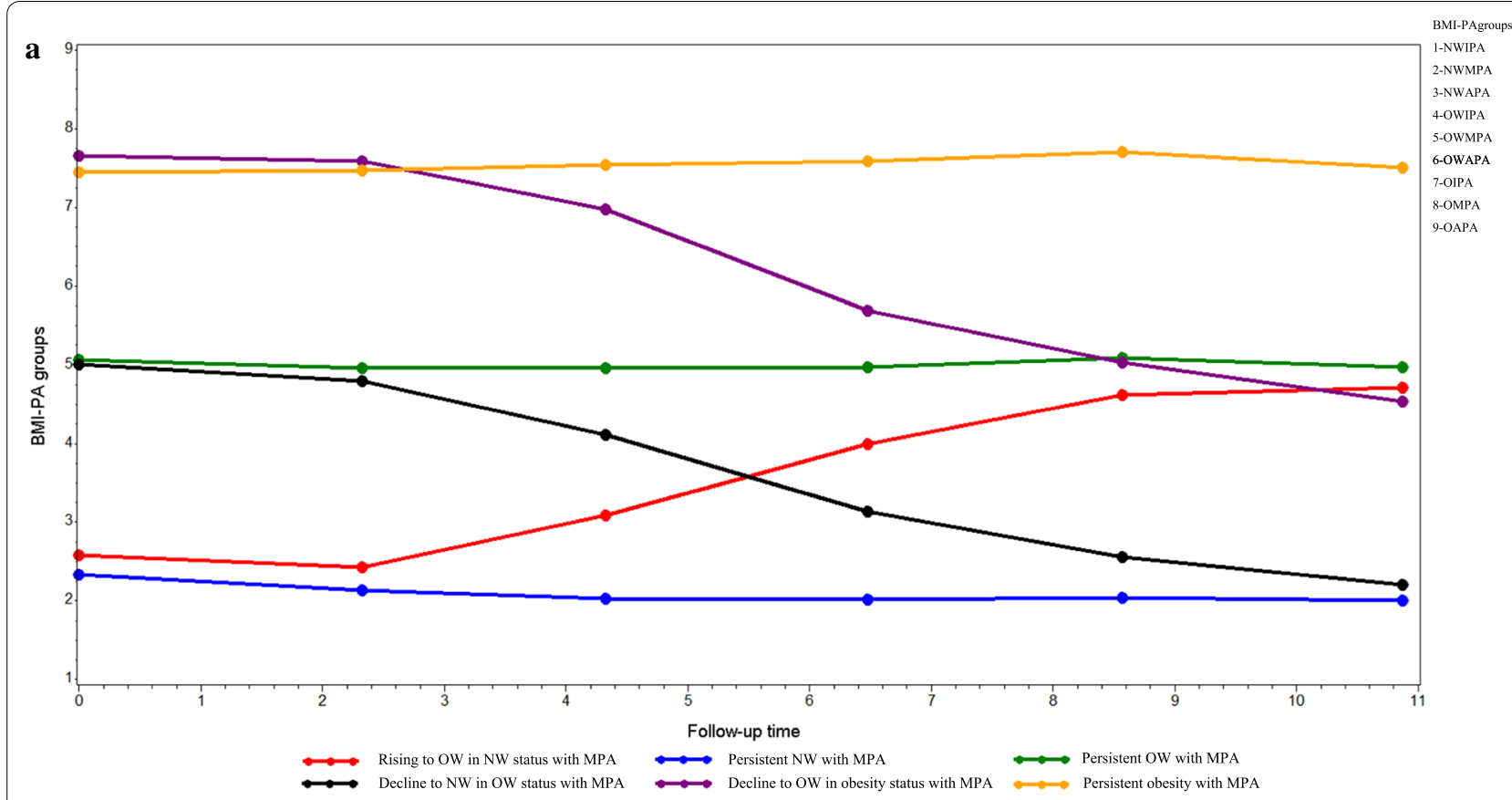

b

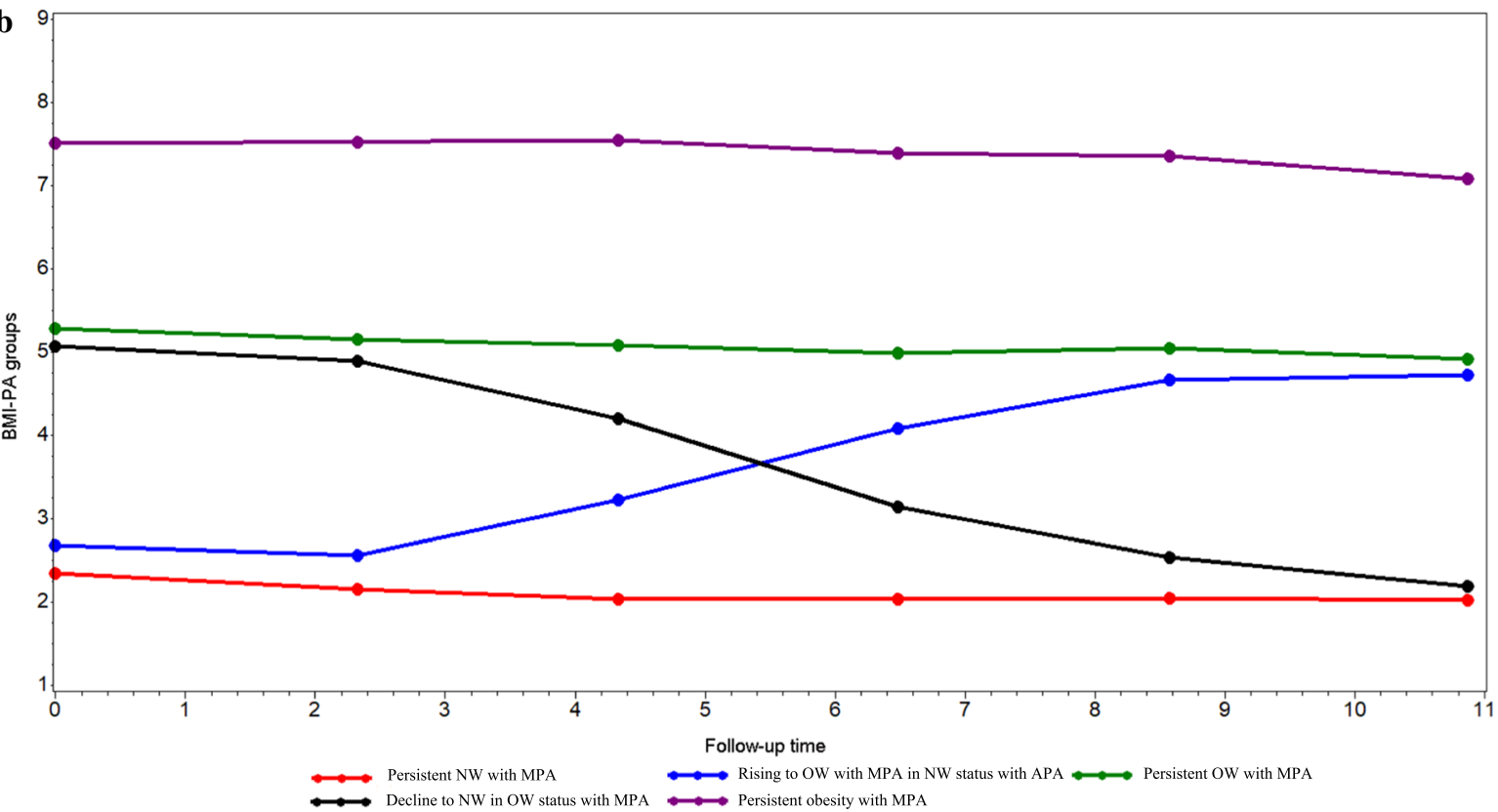

Fig. 2 Trajectory of joint BMI-PA phenotypes during 2006-2016. The joint BMI-PA groups over time for subgroups of participants clustered according to group-based trajectory model (GBTM) estimation are shown. GBTM was produced using Proc Traj procedure in SAS 9.4 software. BMI-PA groups include NWIPA, NWMPA, NWAPA, OWIPA OWMPA, OWAPA, OIPA, OMPA, and OAPA. a Trajectory of joint BMI-PA phenotypes during 2006-2016 for CVDs outcome. b Trajectory of joint BMI-PA phenotypes during 2006-2016 for all-cause mortality outcome. APA active physical activity, BMI body mass index, CVDs cardiovascular diseases, MPA moderate physical activity, NW normal-weight, NWIPA normal weight and inactive physical activity, NWMPA normal weight and moderate physical activity, NWAPA normal weight and active physical activity, OW overweight, OWIPA overweight and inactive physical activity, OWMPA overweight and moderate physical activity, OWAPA overweight and active physical activity, OIPA obesity and inactive physical activity, OMPA obesity and moderate physical activity, OAPA obesity and active physical activity 
groups (Fig. 3). After adjusting covariates, persistent OW with MPA (HR: 1.31, 95\% CI: 1.22-1.41), decline to OW in obesity status with MPA (HR: 1.15, 95\% CI: 1.00-1.32), and persistent obesity with MPA (HR: 1.55, 95\% CI: 1.411.69) were associated with an increased risk of CVDs compared with persistent NW with MPA group (Fig. 4; Table 1). Rising to OW with MPA in NW status with APA (HR: 0.72, 95\% CI: 0.65-0.79), persistent OW with MPA (HR: 0.92, 95\% CI: 0.87-0.97), and decline to NW in OW status with MPA (HR: 0.73, 95\% CI: 0.67-0.80) were associated with a decrease risk of all-cause mortality (Fig. 4; Table 1).

The unadjusted and adjusted cumulative incidence of MI and stroke according to the trajectory groups (Fig. 3). After adjusting covariates, persistent OW with MPA group, decline to OW in obesity status with MPA group, and persistent obesity with MPA group were associated with an increased risk of CVDs compared with persistent NW with MPA group. Different with above results, rising to OW in NW status with MPA group was associated

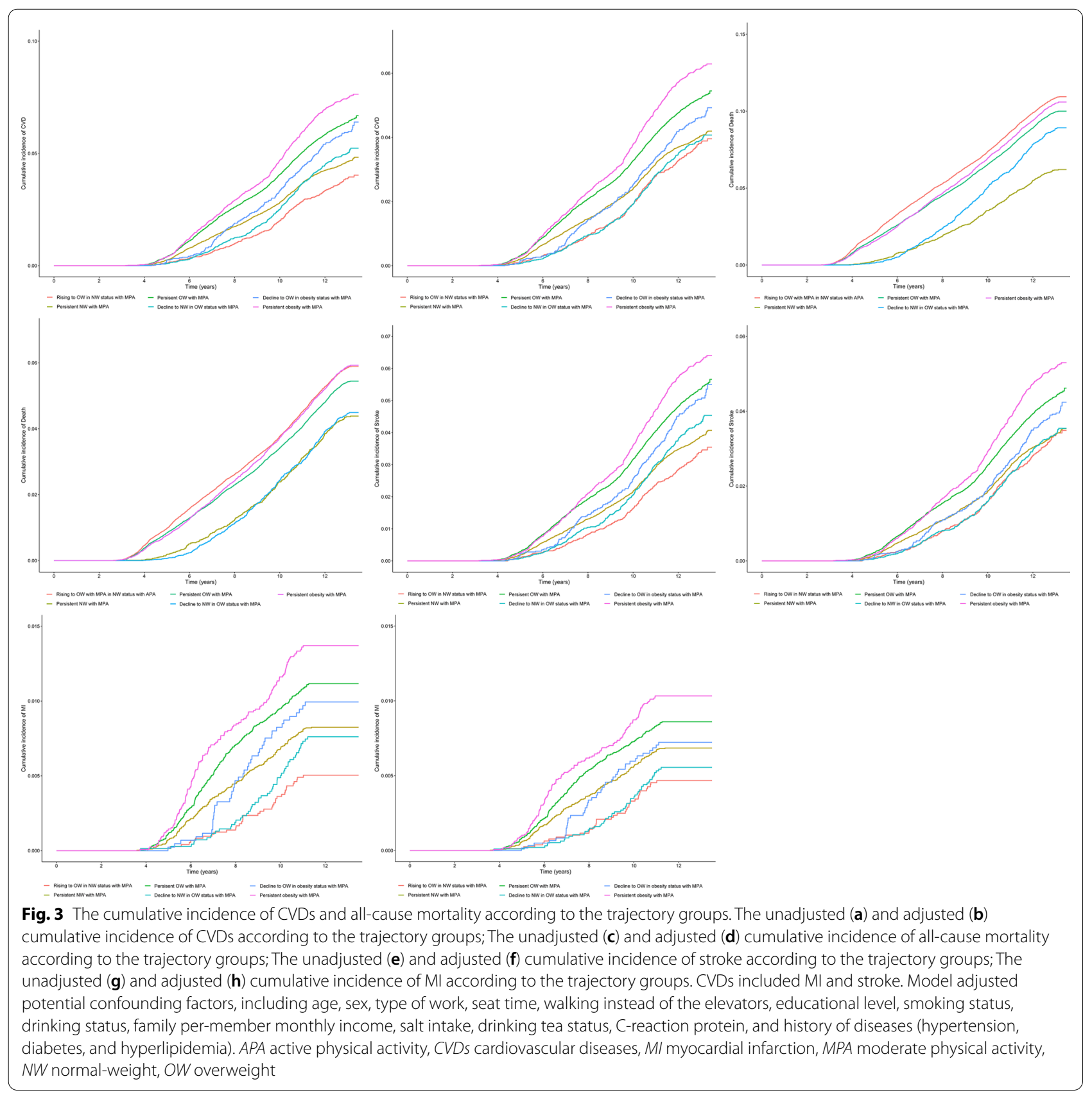




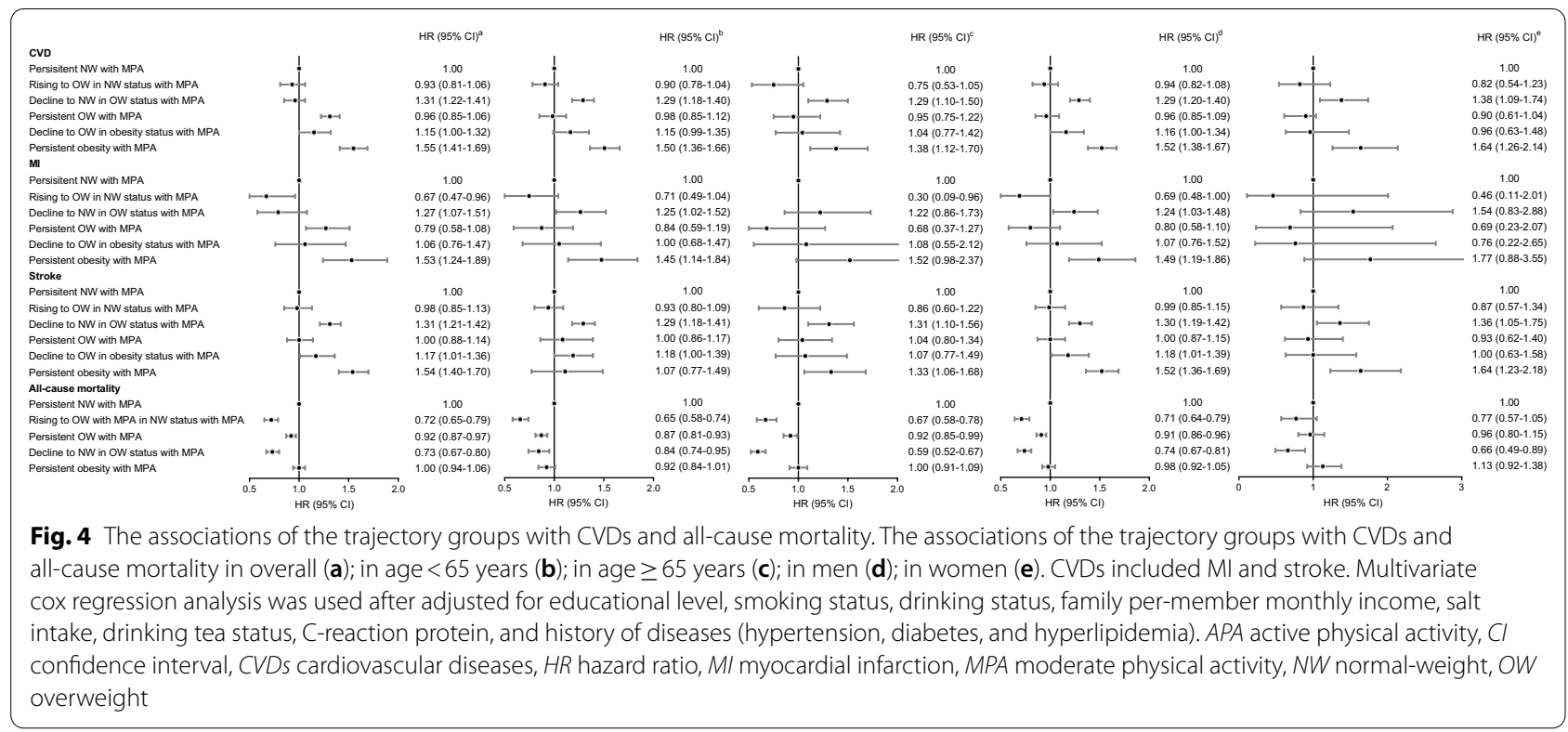

with a decrease risk of MI and persistent obesity with MPA group was not associated with the risk of MI (Fig. 4; Table 1).

\section{Sensitivity analyses and stratification analyses}

Table 2 shows the sensitivity analyses of the association of the trajectory groups with CVDs and all-cause mortality. In the time-depending or competing models, the similar results were obtained.

In the stratified analyses, compared with persistent NW with MPA group, the associations of rising to OW in NW status with MPA group and persistent OW with MPA group with all-cause mortality were stronger in age $\geq 65$ years than in age $<65$ years; however, decline to NW in OW status with MPA group and persistent obesity with MPA group with all-cause mortality were stronger in age $<65$ years than in age $\geq 65$ years (Additional file 1: Table S3). There were significant interactions of age ( $<65$ years old, $\geq 65$ years old) in relationship trajectory groups with all-cause mortality, but no interactions were found for gender in relationship trajectory groups with CVDs, MI, stroke, and all-cause mortality $(P$ interaction $>0.05$ for all) (Additional file 1: Table S3).

\section{Discussion}

In our prospective cohort study of Chinese adults followed biennially from 2006 to 2019, we examined the development of different trajectories of joint phenotypes of BMI and PA during 2006 to 2016, and assessed the associations between these trajectories and risks of CVDs (including MI and stroke) and all-cause mortality. Within the present study, we identified six trajectories and five trajectories using the group-based trajectory analysis for predicting incident CVDs and all-cause mortality, respectively. Compared with persistent NW with MPA group, participants were at $30.8 \%$ and $54.5 \%$ higher risk of CVDs in the persistent OW with MPA and persistent obesity with MPA trajectory group respectively, while at no increased risk of all-cause mortality in all trajectory groups. These findings were overall robust in sensitivity analyses.

Weight trajectory has been focused on multiple studies, is related to the risk of coronary heart disease [30, $31]$, type 2 diabetes [32, 33], blood glucose metabolism [34] and cancers [35]. The present study was the first to assess the links between combined BMI and PA phenotype trajectories with the risk of CVDs and all-cause mortality in Chinese adults. Identifying groups of individuals following similar patterns in distinct trajectories might be useful because it may help to identify different pathways by which OW and obesity development and the mechanisms underlying increasing trends. In addition, this allowed us insight in developing early interventions target specific subgroups based on these distinct trajectories [36].

There was a large cohort study described that waist circumference trajectories (including moderate stable, moderate-high stable and high stable group) were associated with risk of CVDs including stroke and MI [37]. A previously systematic review presented that excess BMI with PA was associated with an increased risk of CVDs, which is consistent with our findings [38]. From the present study, the persistent OW with MPA and persistent obesity with MPA trajectories group were associated 
Table 1 Associations of the trajectory groups with CVDs and all-cause mortality ${ }^{\mathrm{a}}$

\begin{tabular}{|c|c|c|c|c|c|c|c|c|}
\hline & Number $^{b}$ & Rate $(\% o)^{c}$ & $\begin{array}{l}\text { Model 1e } \\
\text { HR }(95 \% \mathrm{Cl})\end{array}$ & $P$ & $\begin{array}{l}\text { Model } 2^{f} \\
\text { HR }(95 \% \text { Cl) }\end{array}$ & $P$ & $\begin{array}{l}\text { Model } 3^{g} \\
\text { HR }(95 \% \mathrm{Cl})\end{array}$ & $P$ \\
\hline \multicolumn{9}{|l|}{$\mathrm{CVDs}^{\mathrm{d}}$} \\
\hline Persistent NW with MPA (reference) & 1208 & 3.81 & - & - & - & - & - & - \\
\hline Rising to OW in NW status with MPA & 275 & 3.20 & $0.83(0.73-0.94)$ & 0.0042 & $0.94(0.82-1.07)$ & 0.3424 & $0.93(0.81-1.06)$ & 0.2701 \\
\hline Persistent OW with MPA & 1836 & 5.33 & $1.40(1.30-1.50)$ & $<0.0001$ & $1.37(1.27-1.47)$ & $<0.0001$ & $1.31(1.22-1.41)$ & $<0.0001$ \\
\hline $\begin{array}{l}\text { Decline to NW in OW status with } \\
\text { MPA }\end{array}$ & 338 & 4.17 & $1.08(0.96-1.22)$ & 0.1977 & $1.00(0.88-1.13)$ & 0.9688 & $0.96(0.85-1.06)$ & 0.5168 \\
\hline $\begin{array}{l}\text { Decline to OW in obesity status with } \\
\text { MPA }\end{array}$ & 252 & 5.00 & $1.30(1.13-1.49)$ & 0.0002 & $1.24(1.08-1.42)$ & 0.0022 & $21.15(1.00-1.32)$ & 0.0494 \\
\hline Persistent obesity with MPA & 843 & 6.23 & $1.64(1.50-1.79)$ & $<0.0001$ & $1.69(1.55-1.85)$ & $<0.0001$ & $1.55(1.41-1.69)$ & $<0.0001$ \\
\hline \multicolumn{9}{|l|}{ Ml } \\
\hline Persistent NW with MPA (reference) & 217 & 3.81 & - & - & - & - & - & - \\
\hline Rising to OW in NW status with MPA & 36 & 3.20 & $0.61(0.43-0.86)$ & 0.0055 & $0.68(0.48-0.98)$ & 0.0366 & $50.67(0.47-0.96)$ & 0.0299 \\
\hline Persistent OW with MPA & 323 & 5.33 & $1.36(1.15-1.62)$ & 0.0005 & $1.33(1.12-1.58)$ & 0.0012 & $2.27(1.07-1.501)$ & 0.0076 \\
\hline $\begin{array}{l}\text { Decline to NW in OW status with } \\
\text { MPA }\end{array}$ & 51 & 4.17 & $0.91(0.67-1.24)$ & 0.5447 & $0.84(0.62-1.14)$ & 0.2594 & $0.79(0.58-1.08)$ & 0.1412 \\
\hline $\begin{array}{l}\text { Decline to OW in obesity status with } \\
\text { MPA }\end{array}$ & 42 & 5.00 & $1.20(0.86-1.67)$ & 0.2764 & $1.15(0.82-1.60)$ & 0.4151 & $1.06(0.76-1.47)$ & 0.7465 \\
\hline Persistent obesity with MPA & 156 & 6.23 & $1.67(1.36-2.05)$ & $<0.0001$ & $1.71(1.39-2.11)$ & $<0.0001$ & $1.53(1.24-1.89)$ & $<0.0001$ \\
\hline \multicolumn{9}{|l|}{ Stroke } \\
\hline Persistent NW with MPA (reference) & 1008 & 3.81 & - & - & - & - & - & - \\
\hline Rising to OW in NW status with MPA & 240 & 3.20 & $0.86(0.75-1.00)$ & 0.0420 & $0.99(0.86-1.13)$ & 0.8327 & $70.98(0.85-1.13)$ & 0.7419 \\
\hline Persistent OW with MPA & 1539 & 5.33 & $1.40(1.29-1.52)$ & $<0.0001$ & $1.37(1.27-1.49)$ & $<0.0001$ & $1.31(1.21-1.42)$ & $<0.0001$ \\
\hline $\begin{array}{l}\text { Decline to NW in OW status with } \\
\text { MPA }\end{array}$ & 291 & 4.17 & $1.12(0.98-1.27)$ & 0.0920 & $1.03(0.91-1.18)$ & 0.6309 & $91.00(0.88-1.14)$ & 0.9831 \\
\hline $\begin{array}{l}\text { Decline to OW in obesity status with } \\
\text { MPA }\end{array}$ & 214 & 5.00 & $1.32(1.14-1.53)$ & 0.0002 & $1.26(1.09-1.46)$ & 0.0022 & $21.17(1.01-1.36)$ & 0.0402 \\
\hline Persistent obesity with MPA & 701 & 6.23 & $1.63(1.48-1.80)$ & $<0.0001$ & $1.68(1.53-1.85)$ & $<0.0001$ & $1.54(1.40-1.70)$ & $<0.0001$ \\
\hline \multicolumn{9}{|l|}{ All-cause mortality } \\
\hline Persistent NW with MPA (reference) & 3072 & 9.35 & - & - & - & - & - & - \\
\hline $\begin{array}{l}\text { Rising to OW with MPA in NW status } \\
\text { with APA }\end{array}$ & 482 & 5.19 & $0.55(0.50-0.60)$ & $<0.0001$ & $0.73(0.66-0.80)$ & $<0.0001$ & $0.72(0.65-0.79)$ & $<0.0001$ \\
\hline Persistent OW with MPA & 3198 & 8.52 & $0.91(0.86-0.95)$ & 0.0001 & $0.95(0.90-1.00)$ & 0.0335 & $0.92(0.87-0.97)$ & 0.0008 \\
\hline $\begin{array}{l}\text { Decline to NW in OW status with } \\
\text { MPA }\end{array}$ & 603 & 7.48 & $0.79(0.73-0.87)$ & $<0.0001$ & $0.75(0.68-0.81)$ & $<0.0001$ & $0.73(0.67-0.80)$ & $<0.0001$ \\
\hline Persistent obesity with MPA & 1499 & 9.02 & $0.96(0.90-1.02)$ & 0.2118 & $1.06(0.99-1.13)$ & 0.0647 & $71.00(0.94-1.06)$ & 0.9347 \\
\hline
\end{tabular}

$A P A$ active physical activity, $C l$ confidence interval, $C R P$ c-reaction protein, $C V D$ cardiovascular disease, $H R$ hazard ratio, $M I$ myocardial infarction, MPA moderate physical activity, NW normal-weight, OW overweight

${ }^{\text {a }}$ Multivariate cox regression analysis was used to evaluate the associations of CVDs and all-cause mortality risk with trajectory groups, adjusting for potential confounding factors

${ }^{b}$ Number represented the number of events

c Per 1,000 person-years

${ }^{\mathrm{d}}$ CVDs included MI and stroke (cerebral infarction, cerebral hemorrhages, and subarachnoid hemorrhage)

e Model 1 was a crude model without adjusted covariates

${ }^{f}$ Model 2 was adjusted for age, sex, type of work, seat time, and walking instead of the elevators

${ }^{9}$ Model 3 was further adjusted for educational level, smoking status, drinking status, family per-member monthly income, salt intake, drinking tea status, CRP, and history of diseases (hypertension, diabetes, and hyperlipidemia)

with an increased risk of CVDs, suggested that persisting OW or obesity could result in CVDs regardless of PA levels. Although PA can have a positive effect on the cardiovascular system [39], moderate exercise intervention may be ineffective in patients with persistent obesity or overweight. A possible reason presented that severe impairment vascular function attributing from persisting obesity or overweight might not be reversed. Similarly, 
Table 2 The sensitivity analyses of the associations of the trajectory groups with CVDs and all-cause mortality ${ }^{\mathrm{a}}$

\begin{tabular}{|c|c|c|c|c|c|c|}
\hline & $\begin{array}{l}\text { Sensitivity } 1^{\text {b }} \\
\text { SHR }(95 \% \mathrm{Cl})\end{array}$ & $P$ & $\begin{array}{l}\text { Sensitivity } 2^{c} \\
\text { HR }(95 \% \mathrm{Cl})\end{array}$ & $P$ & $\begin{array}{l}\text { Sensitivity } 3^{d} \\
\text { SHR }(95 \% C I)\end{array}$ & $P$ \\
\hline \multicolumn{7}{|l|}{$\mathrm{CVDs}^{\mathrm{e}}$} \\
\hline Persistent NW with MPA (reference) & - & - & - & - & & \\
\hline Rising to OW in NW status with MPA & $0.97(0.85-1.11)$ & 0.6513 & $0.91(0.80-1.04)$ & 0.1637 & $0.94(0.83-1.08)$ & 0.3795 \\
\hline Persistent OW with MPA & $1.33(1.24-1.44)$ & $<0.0001$ & $1.33(1.23-1.43)$ & $<0.0001$ & $1.35(1.26-1.46)$ & $<0.0001$ \\
\hline Decline to NW in OW status with MPA & $1.03(0.91-1.16)$ & 0.6518 & $0.97(0.86-1.09)$ & 0.6068 & $1.03(0.91-1.16)$ & 0.6729 \\
\hline Decline to OW in obesity status with MPA & $1.24(1.08-1.42)$ & 0.0024 & $1.16(1.01-1.33)$ & 0.0313 & $1.24(1.08-1.42)$ & 0.0021 \\
\hline Persistent obesity with MPA & $1.55(1.42-1.70)$ & $<0.0001$ & $1.58(1.44-1.73)$ & $<0.0001$ & $1.60(1.46-1.75)$ & $<0.0001$ \\
\hline \multicolumn{7}{|l|}{ Ml } \\
\hline Persistent NW with MPA (reference) & - & - & - & - & & \\
\hline Rising to OW in NW status with MPA & $0.70(0.49-1.00)$ & 0.0524 & $0.69(0.48-0.98)$ & 0.0357 & $0.71(0.50-1.01)$ & 0.0556 \\
\hline Persistent OW with MPA & $1.29(1.08-1.54)$ & 0.0045 & $1.29(1.08-1.53)$ & 0.0046 & $1.31(1.10-1.56)$ & 0.0024 \\
\hline Decline to NW in OW status with MPA & $0.85(0.62-1.16)$ & 0.2915 & $0.81(0.59-1.10)$ & 0.1676 & $0.85(0.63-1.16)$ & 0.3064 \\
\hline Decline to OW in obesity status with MPA & $1.13(0.81-1.58)$ & 0.4580 & $1.06(0.76-1.48)$ & 0.7223 & $1.13(0.81-1.58)$ & 0.4619 \\
\hline Persistent obesity with MPA & $1.54(1.25-1.91)$ & $<0.0001$ & $1.59(1.29-1.96)$ & $<0.0001$ & $1.61(1.30-1.98)$ & $<0.0001$ \\
\hline \multicolumn{7}{|l|}{ Stroke } \\
\hline Persistent NW with MPA (reference) & - & - & - & - & & \\
\hline Rising to OW in NW status with MPA & $1.02(0.89-1.18)$ & 0.7796 & $0.95(0.83-1.10)$ & 0.4892 & $0.99(0.86-1.14)$ & 0.8361 \\
\hline Persistent OW with MPA & $1.34(1.23-1.45)$ & $<0.0001$ & $1.33(1.23-1.44)$ & $<0.0001$ & $1.36(1.25-1.47)$ & $<0.0001$ \\
\hline Decline to NW in OW status with MPA & $1.07(0.94-1.22)$ & 0.3185 & $1.01(0.88-1.15)$ & 0.9416 & $1.07(0.94-1.21)$ & 0.3402 \\
\hline Decline to OW in obesity status with MPA & $1.26(1.09-1.47)$ & 0.0023 & $1.19(1.02-1.38)$ & 0.0234 & $1.27(1.09-1.47)$ & 0.0017 \\
\hline Persistent obesity with MPA & $1.55(1.40-1.72)$ & $<0.0001$ & $1.57(1.43-1.73)$ & $<0.0001$ & $1.59(1.44-1.76)$ & $<0.0001$ \\
\hline \multicolumn{7}{|l|}{ All-cause mortality } \\
\hline Persistent NW with MPA (reference) & - & - & - & - & & \\
\hline Rising to OW with MPA in NW status with APA & - & - & $0.74(0.67-0.81)$ & $<0.0001$ & - & - \\
\hline Persistent OW with MPA & - & - & $0.93(0.88-0.98)$ & 0.0036 & - & - \\
\hline Decline to NW in OW status with MPA & - & - & $0.75(0.69-0.82)$ & $<0.0001$ & - & - \\
\hline Persistent obesity with MPA & - & - & $1.02(0.95-1.08)$ & 0.6192 & - & - \\
\hline
\end{tabular}

${ }^{a}$ Multivariate cox regression analysis was used to evaluate the association of CVDs and all-cause mortality risk with trajectory groups, adjusting for potential confounding factors. HR calculated by cox regression adjusting age, sex, type of work, seat time, walking instead of the elevators, educational level, smoking status, drinking status, family per-member monthly income, salt intake, drinking tea status, CRP, and history of diseases (hypertension, diabetes, and hyperlipidemia)

${ }^{\mathrm{b}}$ Considering non-CVD events death as a competing risk event rather than a censoring event, the association of all-cause mortality risk with trajectory groups was evaluated using competing risk model

'The association of all-cause mortality risk with trajectory groups was evaluated using time-depending model. Age, type of work, seat time, walking instead of the elevators, smoking status, drinking status, family per-member monthly income, salt intake, drinking tea status, CRP, and history of diseases (hypertension, diabetes, and hyperlipidemia) were updated during 2006-2016. Sex and educational level were time-invariant variables

${ }^{\mathrm{d}}$ The association of all-cause mortality risk with trajectory groups was evaluated using time-depending competing risk model

e CVDs included MI and stroke (cerebral infarction, cerebral hemorrhages, and subarachnoid hemorrhage)

APA active physical activity, $C l$ confidence interval, CRP c-reaction protein, CVD cardiovascular disease, HR hazard ratio, MI myocardial infarction, $M P A$ moderate physical activity, NW normal-weight, OW overweight, SHR sub-distribution hazard ratio

persisting at high levels of long-term BMI trajectories was strongly associated with cardio-metabolic traits [40]. Previous study demonstrated that participants with obesity but metabolic healthy $(\mathrm{MH})$ were associated with an increased risk for MI [24] compared with MH-NW, which suggested an important influence of obesity or overweight on CVDs. A possible mechanism also demonstrated that it is difficult to lose fat when we get fatter, because our body makes more strongly energy compensation that burned during PA [41]. Therefore, it is importance to maintain healthy weight for decreasing CVDs risk.

Moreover, the decreased risk of all-cause mortality were observed in rising to OW with MPA in NW status with APA, persistent OW with MPA and decline to NW in OW status with MPA trajectories group, this may suggest the existence of the "obesity paradox" [42]. It is possibility participants with MPA might be a state of MH in the present study, because PA was associated with levels of cardiorespiratory and metabolic fitness [43]. Previous 
studies also presented that participants with $\mathrm{MH}$ and obesity (MHO) or $\mathrm{MH}$ and overweight (MHOW) was not associated with all-cause mortality [44-46], which suggested $\mathrm{MH}$ status might be associated with decrease all-cause mortality. Metabolic phenotypes were associated with body fat distribution patterns including visceral and ectopic fat, which might indirectly result in development of diseases [47]. A possible fat distribution of participants with $\mathrm{MHO}$ or MHOW is a high percentage of gluteofemoral and leg fat mass other than accumulation of visceral fat [48]. Additionally, we previously found that MHOW was the healthiest metabolic phenotype [23], which also suggested the importance of association among metabolic factors, lifestyle factors, and diseases.

Our study is strengthened by the use of large prospective cohort study, including approximately 100,000 participants who were followed-up for more than 13 years, allowed us to perform the joint analyses with sufficient statistical power. The prospective design and high followup rates minimized the potential for recall bias and loss to follow-up. Besides, we firstly evaluated trajectories of joint phenotypes using the GBTM and conducted a series of sensitivity analyses to show the robustness of the findings. The GBTM allows us to identify multi-unobserved subpopulation, and examine differences in change among unobserved subpopulations [27].

There were several limitations about this present study. Firstly, information on PA level was mainly self-reported, thus measurement bias could not be completely avoidable. However, the self-reported PA questionnaire was validated and demonstrated fairly reliability [26, 49]. Secondly, we excluded participants with less than 3 times measurements of joint phenotypes during 2006 to 2016, due to loss to follow-up including occurring outcomes or other reasons, which might be loss information of partial participants, underestimating the effects of trajectories on outcomes. However, we included 98\% of samples in final analysis, and the impact of excluding above participants on the observed association between trajectories and outcomes could be small. Thirdly, we only used BMI to define participants as overweight or obesity. While BMI is widely used in clinical practice, it may be an imperfect measure. And due to the lack of longitudinal measures on indices of fat distribution, we are unable to account for peripheral obesity and abdominal obesity. Fourthly, this study was not a national representative sample, and the racially-homogenous cohort of East Asian ancestry could limit generalizability, the result need to be interpreted cautiously. Fifthly, due to the largest number of participants with MPA, we identified only six and five trajectories of MPA. We cannot estimate the long-term trajectory of obese or overweight patients with high levels of PA and its relationship with CVDs.
Finally, the group-based trajectory analysis identifies the unknown population distribution of trajectories, so the trajectory groups should be considered as clusters of individuals following approximately the same trajectory for homogeneity may be theoretically unrealistic withinclass [28].

\section{Conclusions}

In the large prospective cohort, six long-term trajectories for CVDs and five trajectories for all-cause mortality of joint phenotypes of BMI and PA were identified and were associated with CVDs and all-cause mortality. Participants were at a higher risk of CVDs in the "persistent OW with MPA" and "persistent obesity with MPA" trajectories group. Our results suggested that persisting OW and obesity participants with MPA may not decrease the risk of CVDs in the trajectory group. We additionally observed the decreased risk of all-cause mortality in trajectories group, suggesting the potential existence of "obesity paradox".

\section{Abbreviations}

AvePP: Average posterior probability; AIC: Akakike information criteria; APA: Active physical activity; BIC: Bayesian information criterion; BMl: Body mass index; Cl: Confidence interval; CRP: C-reactive protein; CVD: Cardiovascular disease; FBG: Fasting blood glucose; GBTM: Group-based trajectory model; HC: Hip circumference; HDL-C: High-density lipoprotein cholesterol; HR: Hazard ratio; IQR: Interquartile range; LDL-C: Low-density lipoprotein cholesterol; MI: Myocardial infarction; MPA: Moderate active physical activity; NW: Normal weight; NWAPA: Normal-weight and active physical activity; NWMPA: Normalweight and moderate physical activity; NWIPA: Normal-weight and inactive physical activity; OAPA: Obesity and active physical activity; OIPA: Obesity and inactive physical activity; OMPA: Obesity and moderate physical activity; OW: Overweight; OWAPA: Overweight and active physical activity; OWIPA: Overweight and inactive physical activity; OWMPA: Overweight and moderate physical activity; PA: Physical activity; TC: Total cholesterol; TG: Triglycerides; WC: Waist circumference.

\section{Supplementary Information}

The online version contains supplementary material available at https://doi. org/10.1186/s12967-021-03212-7.

Additional file 1: Table S1. Baseline characteristics of participants according trajectory groups for CVDs outcome. Table S2. Baseline characteristics of participants according trajectory groups for all-cause mortality outcome. Table S3. The association of the trajectory groups with CVDs and all-cause mortality stratified subgroups.

\section{Acknowledgements}

We thank all the research participants in Kailuan study. This work was supported by the China-Australian Collaborative Grant (NSFC 81561128020 NHMRC APP1112767), and the National Natural Science Foundational Research Fund (81561128020). The funders of the study had no role in study design, data collection, data analysis, data interpretation or writing of the report.

\section{Authors' contributions}

YW and SW conceived the study. SC and SW contributed population data resources. QT contributed to the data analysis. BW wrote the original draft. YW 
reviewed and edited manuscript. All authors have read and approved the final manuscript.

\section{Funding}

The study was supported by Grants from the China-Australian Collaborative Grant (NSFC 81561128020-NHMRC APP1112767), and the National Natural Science Foundational Research Fund (81561128020).

\section{Availability of data and materials}

The data underlying this article are available from the corresponding author on reasonable request.

\section{Declarations}

\section{Ethics approval and consent to participate}

This study was approved by the ethics committees of Kailuan General Hospital. Written informed consent form was obtained from all participants.

\section{Consent for publication}

All authors have consented to publication of this research.

\section{Competing interests}

The authors declare that they have no competing interests.

Received: 15 October 2021 Accepted: 20 December 2021

Published online: 28 January 2022

\section{References}

1. Roth GA, Mensah GA, Johnson CO, Addolorato G, Ammirati E, Baddour LM, Barengo NC, Beaton AZ, Benjamin EJ, Benziger CP, et al. Global burden of cardiovascular diseases and risk factors, 1990-2019: update from the GBD 2019 study. J Am Coll Cardiol. 2020;76:2982-3021.

2. GBD 2019 Diseases and Injuries Collaborators. Global burden of 369 diseases and injuries in 204 countries and territories, 1990-2019: a systematic analysis for the Global Burden of Disease Study 2019. Lancet. 2020;396:1204-1222.

3. Zhao D, Liu J, Wang M, Zhang X, Zhou M. Epidemiology of cardiovascular disease in China: current features and implications. Nat Rev Cardiol. 2019;16:203-12.

4. Wu S, Huang Z, Yang X, Zhou Y, Wang A, Chen L, Zhao H, Ruan C, Wu Y, $X$ in $A$, et al. Prevalence of ideal cardiovascular health and its relationship with the 4-year cardiovascular events in a northern Chinese industrial city. Circ Cardiovasc Qual Outcomes. 2012;5:487-93.

5. Zhai F, Wang H, Du S, He Y, Wang Z, Ge K, Popkin BM. Prospective study on nutrition transition in China. Nutr Rev. 2009;67(Suppl 1):S56-61.

6. Kivimäki M, Kuosma E, Ferrie JE, Luukkonen R, Nyberg ST, Alfredsson L, Batty GD, Brunner EJ, Fransson E, Goldberg M, et al. Overweight, obesity, and risk of cardiometabolic multimorbidity: pooled analysis of individual-level data for 120813 adults from 16 cohort studies from the USA and Europe. Lancet Public Health. 2017;2:e277-285.

7. Khan SS, Ning H, Wilkins JT, Allen N, Carnethon M, Berry JD, Sweis RN, Lloyd-Jones DM. Association of body mass index with lifetime risk of cardiovascular disease and compression of morbidity. JAMA Cardiol. 2018;3:280-7.

8. Flegal KM, Kit BK, Orpana H, Graubard BI. Association of all-cause mortality with overweight and obesity using standard body mass index categories: a systematic review and meta-analysis. JAMA. 2013;309:71-82.

9. Aune D, Sen A, Prasad M, Norat T, Janszky I, Tonstad S, Romundstad P, Vatten $\sqcup$. BMI and all cause mortality: systematic review and non-linear dose-response meta-analysis of 230 cohort studies with 3.74 million deaths among 3.03 million participants. BMJ. 2016;353:i2156.

10. Romero-Corral A, Montori VM, Somers VK, Korinek J, Thomas RJ, Allison TG, Mookadam F, Lopez-Jimenez F. Association of bodyweight with total mortality and with cardiovascular events in coronary artery disease: a systematic review of cohort studies. Lancet. 2006;368:666-78.
11. Banack HR, Kaufman JS. The obesity paradox: understanding the effect of obesity on mortality among individuals with cardiovascular disease. Prev Med. 2014;62:96-102.

12. Bucholz EM, Rathore SS, Reid KJ, Jones PG, Chan PS, Rich MW, Spertus JA, Krumholz HM. Body mass index and mortality in acute myocardial infarction patients. Am J Med. 2012;125:796-803.

13. Ferreira I, Stehouwer CD. Obesity paradox or inappropriate study designs? Time for life-course epidemiology. J Hypertens. 2012;30:2271-5.

14. Myers J, McAuley P, Lavie CJ, Despres JP, Arena R, Kokkinos P. Physical activity and cardiorespiratory fitness as major markers of cardiovascular risk: their independent and interwoven importance to health status. Prog Cardiovasc Dis. 2015:57:306-14.

15. Lee IM, Shiroma EJ, Lobelo F, Puska P, Blair SN, Katzmarzyk PT. Effect of physical inactivity on major non-communicable diseases worldwide: an analysis of burden of disease and life expectancy. Lancet. 2012;380:219-29.

16. Koolhaas CM, Dhana K, Schoufour JD, Ikram MA, Kavousi M, Franco $\mathrm{OH}$. Impact of physical activity on the association of overweight and obesity with cardiovascular disease: the Rotterdam Study. Eur J Prev Cardiol. 2017;24:934-41.

17. Zhang X, Cash RE, Bower JK, Focht BC, Paskett ED. Physical activity and risk of cardiovascular disease by weight status among U.S adults. PLoS ONE. 2020;15:e0232893.

18. Valenzuela PL, Santos-Lozano A, Barrán AT, Fernández-Navarro P, Castillo-García A, Ruilope LM, Ríos Insua D, Ordovas JM, Ley V, Lucia A. Joint association of physical activity and body mass index with cardiovascular risk: a nationwide population-based cross-sectional study. Eur J Prev Cardiol. 2021.

19. Sanchez-Lastra MA, Ding D, Dalene KE, Ekelund U, Tarp J. Physical activity and mortality across levels of adiposity: a prospective cohort study from the UK Biobank. Mayo Clin Proc. 2021;96:105-19.

20. Carlsson AC, Ärnlöv J, Sundström J, Michaëlsson K, Byberg L, Lind L. Physical activity, obesity and risk of cardiovascular disease in middleaged men during a median of 30 years of follow-up. Eur J Prev Cardiol. 2016;23:359-65.

21. Jia Z, Zhou Y, Liu X, Wang Y, Zhao X, Wang Y, Liang W, Wu S. Comparison of different anthropometric measures as predictors of diabetes incidence in a Chinese population. Diabetes Res Clin Pract. 2011;92:265-71.

22. Wang A, Zhang Y, Li J, Zhao Q, Cao Y, Li J, Zhang R, Chen S, Gao J, Wu S. High SBP trajectories are associated with risk of all-cause death in general Chinese population. J Hypertens. 2018;36:1299-305.

23. Tian Q, Wang A, Zuo Y, Chen S, Hou H, Wang W, Wu S, Wang Y. All-cause mortality in metabolically healthy individuals was not predicted by overweight and obesity. JCI Insight. 2020;5:e136982.

24. Xu Y, Li H, Wang A, Su Z, Yang G, Luo Y, Tao L, Chen S, Wu S, Wang Y, et al. Association between the metabolically healthy obese phenotype and the risk of myocardial infarction: results from the Kailuan study. Eur J Endocrinol. 2018;179:343-52.

25. Chen C, Lu FC; Department of Disease Control Ministry of Health, PR China. The guidelines for prevention and control of overweight and obesity in Chinese adults. Biomed Environ Sci. 2004;17(Suppl):1-36.

26. Yu Y, Dong Z, Li Y, Zhang J, Yin S, Gao X, Wu S. The cardiovascular and cerebrovascular health in North China From 2006 to 2011: results from the KaiLuan Study. Front Cardiovasc Med. 2021;8:683416.

27. Nagin DS. Group-based trajectory modeling: an overview. Ann Nutr Metab. 2014:65:205-10

28. Nagin DS, Odgers CL. Group-based trajectory modeling in clinical research. Annu Rev Clin Psychol. 2010;6:109-38.

29. Nagin DS, Jones BL, Passos VL, Tremblay RE. Group-based multi-trajectory modeling. Stat Methods Med Res. 2018;27:2015-23.

30. Willett WC, Manson JE, Stampfer MJ, Colditz GA, Rosner B, Speizer FE, Hennekens $\mathrm{CH}$. Weight, weight change, and coronary heart disease in women. Risk within the 'normal' weight range. JAMA. 1995;273:461-5.

31. Wang X, Dong B, Huang S, Ma Y, Zou Z, Ma J, Yang Z, Wang Z. Body mass index trajectory and incident hypertension: results from a longitudinal cohort of Chinese Children and Adolescents, 2006-2016. Am J Public Health. 2020;110:1689-95. 
32. Nano J, Dhana K, Asllanaj E, Sijbrands E, Ikram MA, Dehghan A, Muka T, Franco $\mathrm{OH}$. Trajectories of BMI before diagnosis of type 2 diabetes: the Rotterdam Study. Obesity (Silver Spring). 2020;28:1149-56.

33. Luo J, Hodge A, Hendryx M, Byles JE. BMl trajectory and subsequent risk of type 2 diabetes among middle-aged women. Nutr Metab Cardiovasc Dis. 2021;31:1063-70.

34. Walsh El, Shaw J, Cherbuin N. Trajectories of BMI change impact glucose and insulin metabolism. Nutr Metab Cardiovasc Dis. 2018;28:243-51.

35. Liu Y, Warren Andersen S, Wen W, Gao YT, Lan Q, Rothman N, Ji BT, Yang G, Xiang YB, Shu XO, et al. Prospective cohort study of general and central obesity, weight change trajectory and risk of major cancers among Chinese women. Int J Cancer. 2016;139:1461-70.

36. Mattsson M, Maher GM, Boland F, Fitzgerald AP, Murray DM, Biesma R. Group-based trajectory modelling for BMI trajectories in childhood: a systematic review. Obes Rev. 2019;20:998-1015.

37. Wang L, Lee Y, Wu Y, Zhang X, Jin C, Huang Z, Wang Y, Wang Z, Kris-Etherton $\mathrm{P}, \mathrm{Wu} \mathrm{S}$, et al. A prospective study of waist circumference trajectories and incident cardiovascular disease in China: the Kailuan Cohort Study. Am J Clin Nutr. 2021;113:338-47.

38. Fogelholm M. Physical activity, fitness and fatness: relations to mortality, morbidity and disease risk factors. A systematic review. Obes Rev. 2010;11:202-21.

39. Lavie CJ, Arena R, Swift DL, Johannsen NM, Sui X, Lee DC, Earnest CP, Church TS, O'Keefe JH, Milani RV, et al. Exercise and the cardiovascular system: clinical science and cardiovascular outcomes. Circ Res. 2015;117:207-19.

40. Buscot MJ, Thomson RJ, Juonala M, Sabin MA, Burgner DP, Lehtimäki T, Hutri-Kähönen N, Viikari JSA, Raitakari OT, Magnussen CG. Distinct childto-adult body mass index trajectories are associated with different levels of adult cardiometabolic risk. Eur Heart J. 2018;39:2263-70.

41. Careau V, Halsey LG, Pontzer H, Ainslie PN, Andersen LF, Anderson $L$, Arab L, Baddou I, Bedu-Addo K, Blaak EE, et al. Energy compensation and adiposity in humans. Curr Biol. 2021;31:4659-4666.e4652.

42. Lavie CJ, Sharma A, Alpert MA, De Schutter A, Lopez-Jimenez F, Milani RV, Ventura HO. Update on obesity and obesity paradox in heart failure. Prog Cardiovasc Dis. 2016:58:393-400.

43. Stamatakis E, Gale J, Bauman A, Ekelund U, Hamer M, Ding D. Sitting time, physical activity, and risk of mortality in adults. J Am Coll Cardiol. 2019;73:2062-72.

44. Zheng R, Zhou D, Zhu Y. The long-term prognosis of cardiovascular disease and all-cause mortality for metabolically healthy obesity: a systematic review and meta-analysis. J Epidemiol Community Health. 2016;70:1024-31.

45. Hamer M, Johnson W, Bell JA. Improving risk estimates for metabolically healthy obesity and mortality using a refined healthy reference group. Eur J Endocrinol. 2017;177:169-74

46. Jae SY, Kurl S, Fernhall B, Kunutsor SK, Franklin BA, Laukkanen JA. Are metabolically healthy overweight/obese men at increased risk of sudden cardiac death? Mayo Clin Proc. 2018:93:1266-70.

47. Kramer CK, Zinman B, Retnakaran R. Are metabolically healthy overweight and obesity benign conditions?: A systematic review and metaanalysis. Ann Intern Med. 2013;159:758-69.

48. Stefan N, Schick F, Häring HU. Causes, characteristics, and consequences of metabolically unhealthy normal weight in humans. Cell Metab. 2017:26:292-300.

49. Wolf AM, Hunter DJ, Colditz GA, Manson JE, Stampfer MJ, Corsano KA, Rosner B, Kriska A, Willett WC. Reproducibility and validity of a self-administered physical activity questionnaire. Int J Epidemiol. 1994;23:991-9.

\section{Publisher's Note}

Springer Nature remains neutral with regard to jurisdictional claims in published maps and institutional affiliations.

Ready to submit your research? Choose BMC and benefit from:

- fast, convenient online submission

- thorough peer review by experienced researchers in your field

- rapid publication on acceptance

- support for research data, including large and complex data types

- gold Open Access which fosters wider collaboration and increased citations

- maximum visibility for your research: over $100 \mathrm{M}$ website views per year

At $\mathrm{BMC}$, research is always in progress.

Learn more biomedcentral.com/submissions 